\title{
Long Time Behavior of the Solution of the Two-Dimensional Dissipative QGE in Lei-Lin Spaces
}

\author{
Moez Benhamed (iD ${ }^{1,2}$ and Sahar Mohammad Abusalim ${ }^{1}$ \\ ${ }^{1}$ Department of Mathematics, College of Sciences and Arts in Gurayat, Jouf University, Sakakah, Saudi Arabia \\ ${ }^{2}$ Department of Mathematics, Faculty of Sciences of Tunis, University of Tunis El Manar, Tunis, LR03ES04, Tunisia \\ Correspondence should be addressed to Moez Benhamed; moez.benhamed@fst.utm.tn
}

Received 15 December 2019; Accepted 21 March 2020; Published 29 April 2020

Academic Editor: Vladimir V. Mityushev

Copyright (c) 2020 Moez Benhamed and Sahar Mohammad Abusalim. This is an open access article distributed under the Creative Commons Attribution License, which permits unrestricted use, distribution, and reproduction in any medium, provided the original work is properly cited.

In this paper, we study the asymptotic behavior of the two-dimensional quasi-geostrophic equations with subcritical dissipation. More precisely, we establish that $\|\theta(t)\|_{\mathscr{X}^{1-2 \alpha}}$ vanishes at infinity.

\section{Introduction and Statement of Main Results}

In this paper, we consider the initial value problem for the 2D quasi-geostrophic equations with subcritical dissipation $(\mathrm{QG})_{\alpha}$ :

$$
(\mathrm{QG})_{\alpha} \quad\left\{\begin{array}{l}
\partial_{t} \theta+u \cdot \nabla \theta+k \Lambda^{2 \alpha} \theta=0, \quad x \in \mathbb{R}^{2}, t>0 \\
u=R^{\perp} \theta=\left(-\mathfrak{R}_{2} \theta, \Re_{1} \theta\right) \\
\theta(0, x)=\theta^{0}(x)
\end{array}\right.
$$

where $1 / 2<\alpha \leq 1$ is a real number and $k>0$ is a dissipative coefficient. $\Lambda$ is the operator defined by the fractional power of $-\Delta$ :

$$
\begin{array}{r}
\Lambda=(-\Delta)^{1 / 2}, \\
\widehat{\Lambda g}=\left(-\widehat{\Delta)^{1 / 2}} g=|\xi| \widehat{g},\right.
\end{array}
$$

and more generally

$$
\widehat{\Lambda^{2 \alpha} g}=\left(-\widehat{\Delta)^{\alpha}} g=|\xi|^{2 \alpha} \widehat{g}\right.
$$

where $\hat{g}(\xi)$ denotes the Fourier transform of $g . \theta(x, t)$ is an unknown scalar function representing potential temperature, and $u=\left(u_{1}, u_{2}\right)$ is the divergence free velocity which is determined by the Riesz transformation of $\theta$ in the following way:

$$
\begin{aligned}
& u_{1}=-\Re_{2} \theta=-\partial_{2}(-\Delta)^{-1 / 2} \theta, \\
& u_{2}=-\mathfrak{R}_{1} \theta=-\partial_{1}(-\Delta)^{-1 / 2} \theta
\end{aligned}
$$

Let us fix $k=1$ for the rest of the paper.

The $2 D$ quasi-geostrophic fluid is an important model in geophysical fluid dynamics, which are special cases of the general quasi-geostrophic approximations for atmospheric and oceanic fluid flow with the small local Rossby number which ensures the validity of the geostrophic balance between the pressure gradient and the Coriolis force (see [1]). Furthermore, this quasi-geostrophic fluid motion equation shares many features with fundamental fluid motion equations. When $k=0$, this equation is comparable to the vorticity formulation of the Euler equations (see [2]). (QG) with $\alpha=1 / 2$ shares similar features with the three-dimensional Navier-Stokes equations. Thus, $\alpha=1 / 2$ is therefore referred as the critical case, while the cases $\alpha=1 / 2$ and $\alpha=$ $1 / 2$ are subcritical and supercritical, respectively.

The existence of a global weak solution was established by several researchers. The reader is referred to [3-7] and their references. Furthermore, in the subcritical case, Constantin and $\mathrm{Wu}$ [8] proved that every sufficiently smooth initial data give rise to a unique global smooth solution. For the critical case, $\alpha=1 / 2$, Constantin et al. [9] proved that there exists of a unique global classical solution for any small initial data in $L^{\infty}$. The hypothesis requiring smallness in $L^{\infty}$ was removed by Caffarelli and Vasseur [10] 
and Dong and $\mathrm{Du}$ [5]. In [7], the authors proved persistence of a global solution in $\mathscr{C}^{\infty}$ for to any $\mathscr{C}^{\infty}$ periodic initial data. Chae and Lee [6] established the global existence and uniqueness of solution for any small initial data in the Besov space $\dot{B}_{2,1}^{2-2 \alpha}$.

The global existence for the quasi-geostrophic equation has been studied in the previous work of Benameur and Benhamed [3]. The authors have introduced new spaces $\mathscr{X}^{1-2 \alpha}\left(\mathbb{R}^{2}\right)$ defined as follows:

$$
\begin{gathered}
\mathscr{X}^{1-2 \alpha}\left(\mathbb{R}^{2}\right)=\left\{f \in \mathrm{S}^{\prime}\left(\mathbb{R}^{2}\right) ;\left(\xi \mapsto|\xi|^{1-2 \alpha} \widehat{f}(\xi)\right) \in L^{1}\left(\mathbb{R}^{2}\right)\right\}, \\
\frac{1}{2}<\alpha \leq 1,
\end{gathered}
$$

which is equipped with the norm

$$
\|f\|_{X^{1-2 \alpha}}=\int_{\mathbb{R}^{2}}|\xi|^{1-2 \alpha}|\widehat{f}(\xi)| \mathrm{d} \xi .
$$

More precisely, their result is as follows..

Theorem 1. Let $\theta^{0} \in \mathscr{X}^{1-2 \alpha}\left(\mathbb{R}^{2}\right)$. There is a time $T>0$ and unique solution $\theta \in \mathscr{C}\left([0, T], \mathscr{X}^{1-2 \alpha}\left(\mathbb{R}^{2}\right)\right)$ of $(Q G)_{\alpha}$; moreover, $\theta \in L^{1}\left([0, T], \mathscr{X}^{1}\left(\mathbb{R}^{2}\right)\right)$.

If $\left\|\theta^{0}\right\|_{\mathscr{X}^{1-2 \alpha}}<1 / 4$, then the solution is global and

$$
\begin{aligned}
& \|\theta(t)\|_{\mathscr{X}^{1-2 \alpha}}+\left(1-4\left\|\theta^{0}\right\|_{\mathscr{X}^{1-2 \alpha}}\right) \int_{0}^{t}\|\theta(s)\|_{\mathscr{X}^{1}} \mathrm{~d} s \leq\left\|\theta^{0}\right\|_{X^{1-2 \alpha}}, \\
& \forall t \geq 0
\end{aligned}
$$

We will recall further that the paper titled "Behavior of solutions of 2D quasi-geostrophic equations" by Constantin and $\mathrm{Wu}$ [8] (published in the SIAM Journal of Mathematical Analysis 30, 1999) has several results along the same lines. In particular, it includes the following result.

Theorem 2. Let $0<\alpha \leq 1 / 2$ and $\theta_{0} \in L^{1}\left(\mathbb{R}^{2}\right) \cap L^{2}\left(\mathbb{R}^{2}\right)$. Then, there exists a weak solution $\theta$ of the $(Q G)_{\alpha}$ equation with initial data $\theta_{0}$ such that

$$
\|\theta(., t)\|_{L^{2}\left(\mathbb{R}^{2}\right)} \leq C(1+t)^{-(1 / 2 \alpha)},
$$

Proof. where $C$ is a constant depending on $L^{1}$ and $L^{2}$ norms of $\theta_{0}$.

The decay of $L^{2}$ and Sobolev norms and asymptotic behaviour of solutions to the quasi-geostrophic equations have also been addressed in many articles (see, for example, $[5,11-22]$

Global-in-time well-posedness, time-decay, and asymptotic behavior of solutions are core properties in understanding how fluid mechanics models work. In fact, there is a rich literature about those properties for fluid dynamics PDEs via several approaches and different frameworks. In this direction, there are studies in frameworks containing singular data and invariant under the scaling (critical spaces), where the smallness conditions are taken in the weak norms of the critical spaces (e.g., see the review book $[23,24]$ ). Of particular interest is the analysis of PDEs in critical frameworks whose structure is based on the Fourier transform. Navier-Stokes and quasi-geostrophic equations have been studied in several spaces such as $\operatorname{PM}^{a}[13,25,26]$, Fourier-Besov $\mathscr{F}_{q, r}^{2-(3 / q)}[7,27,28]$, Lei-Lin spaces $\mathscr{X}^{\sigma}$ $[3,29]$, and Fourier-Besov-Morrey $\mathscr{F} \mathrm{N}_{p, \mu, r}^{s}[11,30]$. In the case $q=1$, Fourier-Besov spaces $\mathscr{F}_{\mathscr{B}_{q, r}^{s}}\left(\mathbb{R}^{n}\right)$ were introduced by Iwabuchi [31] in the context of parabolic-elliptic Keller-Segel system. Later, Iwabuchi and Takada [7] used critical $\mathscr{F} \mathscr{B}_{1,2}^{-1}$-spaces in order to obtain a global wellposedness class (uniformly with respect to the angular velocity) for the Navier-Stokes-Coriolis system. Taking in particular $\Omega=0$, they also obtained a global well-posedness result for the $3 D$ Navier-Stokes equations with small initial data in $\dot{\mathscr{F}} \mathscr{B}_{1,2}^{-1}$. Konieczny and Yoneda [28] also showed global well-posedness and asymptotic stability of small solutions for $3 D$ Navier-Stokes equations (and Navier-Stokes-Coriolis) in critical Fourier-Besov

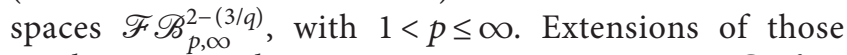
results to critical Fourier-Besov-Morrey spaces $\mathscr{F} \mathrm{N}_{p, \mu, r}^{s}$ (larger than $\dot{\mathscr{F}} \mathscr{B}_{p, r}^{s}$ ) were obtained in $[30,32] \stackrel{p, \mu, r}{\text { for }}$ Navier-Stokes equations (and Navier-Stokes-Coriolis) and active scalar equations with fractional dissipation $(-\Delta)^{\alpha}$ (including the $2 D$ quasi-geostrophic equation $\left.(\mathrm{QG})_{\alpha}\right)$, respectively.

The main goal of this paper is to study the $2 D$ quasigeostrophic equation in the framework of critical Lei-Lin spaces $\mathscr{X}^{\sigma}$ with $\sigma=1-2 \alpha$ and $2 / 3<\alpha \leq 1$. We show that solution $\theta$ presents the asymptotic property $\|\theta(t)\|_{X^{1-2 \alpha}} \longrightarrow 0$ as $t \longrightarrow 0$ provided that $\|\theta(t)\|_{X^{1-2 \alpha}}<1 / 4$. For that, we use standard interpolation in the Fourier space, energy estimates in $L^{2}$, and Young's inequality of convolutions, among others. Our main result is the following.

Theorem 3. Let $2 / 3<\alpha<1,\left\|\theta^{0}\right\|_{X^{1-2 \alpha}}<1 / 4$ and $\theta \in \mathscr{C}\left(\mathbb{R}^{+}, \mathscr{X}^{1-2 \alpha}\left(\mathbb{R}^{2}\right)\right)$ be a global solution of $(Q G)_{\alpha}$ given by Theorem 1. Then,

$$
\lim _{t \rightarrow \infty}\|\theta(t)\| x^{1-2 \alpha}=0 .
$$

Remark 1. Remark that our main result is not implied by Theorem 2 of Constantin- Wu, because our works concern the asymptotic behavior of solution in the Lei-Lin space who belongs to a class whose definition of the norm is based on Fourier transform, but it is not contained in $L^{2}$ while that the result of Theorem 2 concerns the study in the space $L^{1} \cap L^{2}$. The proof techniques (for Theorem 3 and Lemma 1) appeal to fairly standard interpolation in the Fourier space (which creates the need for $\alpha>2 / 3$ instead of the more natural $\alpha>1 / 2$ ), energytype $L^{2}$ estimates for $\theta_{n}$ that exploit the natural appearance of the $x^{-1}$-norm from Young's inequality of convolutions, and two uses of (Theorem 3) (proved in the earlier work).

The remaining part of the paper is organized as follows. The main results are given in Section 1. We explain the framework in Section 2. The long time behaviour (Theorem 3) is established in Section 3. 


\section{Preliminary}

Let us recall that in [29], Lei and Lin introduced a new space, named Lei-Lin space $\mathscr{X}^{-1}$, which belongs to a class whose definition of norm is based on the Fourier transform but is not contained in $L^{2}$. In [3], Benameur and Benhamed defined the spaces that are useful for the study of well-posedness of PDEs of parabolic, elliptic, and dispersive types. More precisely, for $\sigma \in \mathbb{R}$, we define

$$
\begin{aligned}
\mathscr{X}^{\sigma}\left(\mathbb{R}^{2}\right)= & \left\{f \in \mathrm{S}^{\prime}\left(\mathbb{R}^{2}\right) ; \hat{f} \in L_{\mathrm{loc}}^{1}\left(\mathbb{R}^{2}\right) \text { and }\left(\xi \mapsto|\xi|^{\sigma} \widehat{f}(\xi)\right)\right. \\
& \left.\in L^{1}\left(\mathbb{R}^{2}\right)\right\},
\end{aligned}
$$

equipped with the norm

$$
\|f\|_{\chi^{\sigma}}=\int_{\mathbb{R}^{2}}|\xi|^{\sigma}|\widehat{f}(\xi)| \mathrm{d} \xi
$$

To prove Theorem 3, we need the following lemma.

Lemma 1. For $2 / 3<\alpha<1, \dot{H}^{\alpha}\left(\mathbb{R}^{2}\right) \longrightarrow \mathscr{X}^{1-2 \alpha}\left(\mathbb{R}^{2}\right)$. More precisely, if $f \in \dot{H}^{\alpha}\left(\mathbb{R}^{2}\right) \cap L^{2}\left(\mathbb{R}^{2}\right)$. Then, we have

$$
\|f\|_{\mathscr{X}^{1-2 \alpha}} \leq C\|f\|_{L^{2}}^{((3 \alpha-2) / \alpha)}\|f\|_{\dot{H}^{\alpha}}^{((2-2 \alpha) / \alpha)} .
$$

Proof. For $\lambda>0$, put $\|f\|_{\mathscr{X}^{1-2 \alpha}}=\mathbf{A}_{\lambda}+\mathbf{B}_{\lambda}$, where

$$
\begin{aligned}
& \mathbf{A}_{\lambda}=\int_{|\xi|<\lambda}|\xi|^{1-2 \alpha}|\widehat{f}(\xi)| \mathrm{d} \xi, \\
& \mathbf{B}_{\lambda}=\int_{|\xi|<\lambda}|\xi|^{1-2 \alpha}|\widehat{f}(\xi)| \mathrm{d} \xi .
\end{aligned}
$$

Let us start by controlling the first term; using Cauchy-Schwarz inequality, we get

$$
\begin{aligned}
\mathbf{A}_{\lambda} & =\int_{|\xi|<\lambda}|\xi|^{1-2 \alpha} \times|\widehat{f}(\xi)| \mathrm{d} \xi \\
& \leq\left(\int_{|\xi|<\lambda}|\widehat{f}(\xi)| \mathrm{d} \xi\right)^{1 / 2}\left(\int_{|\xi|<\lambda}|\xi|^{2-4 \alpha} \mathrm{d} \xi\right)^{1 / 2} \\
& \leq \sqrt{4 \pi}\|f\|_{L^{2}}\left(\int_{0}^{\lambda} r^{3-4 \alpha} \mathrm{d} r\right)^{1 / 2} \\
& \leq \sqrt{\frac{4 \pi}{4-4 \alpha}}\|f\|_{L^{2}}\left(\lambda^{4-4 \alpha}\right)^{1 / 2} .
\end{aligned}
$$

Therefore,

$$
\mathbf{A}_{\lambda} \leq \sqrt{\frac{4 \pi}{4-4 \alpha}} \lambda^{2-2 \alpha}\|f\|_{L^{2}} .
$$

A similar calculation to the foregoing yields

$$
\begin{aligned}
\mathbf{B}_{\lambda} & =\int_{|\xi|>\lambda}|\xi|^{\alpha}|\widehat{f}(\xi)| \times|\xi|^{1-3 \alpha} \mathrm{d} \xi \\
& \leq\left(\int_{|\xi|>\lambda}|\xi|^{2 \alpha}|\widehat{f}(\xi)| \mathrm{d} \xi\right)^{1 / 2}\left(\int_{|\xi|>\lambda}|\xi|^{2-6 \alpha} \mathrm{d} \xi\right)^{1 / 2} \\
& \leq \sqrt{4 \pi}\|f\|_{\dot{H}^{\alpha}}\left(\int_{\lambda}^{\infty} r^{3-6 \alpha} \mathrm{d} r\right)^{1 / 2} \\
& \leq \sqrt{\frac{4 \pi}{6 \alpha-4}} \lambda^{2-3 \alpha}\|f\|_{\dot{H}^{\alpha}} .
\end{aligned}
$$

Then,

$$
\mathbf{B}_{\lambda} \leq \sqrt{\frac{4 \pi}{6 \alpha-4}} \lambda^{2-3 \alpha}\|f\|_{\dot{H}^{\alpha}}
$$

Combining (15) and (17), we get

$$
\|f\|_{\mathscr{X}^{1-2 \alpha}} \leq \sqrt{\frac{4 \pi}{4-4 \alpha}} \lambda^{2-2 \alpha}\|f\|_{L^{2}}+\sqrt{\frac{4 \pi}{6 \alpha-4}} \lambda^{2-3 \alpha}\|f\|_{\dot{H}^{\alpha}} .
$$

Optimizing $\|f\|_{\mathscr{X}^{1-2 \alpha}}$, it suffices to choose $\lambda=\left(\|f\|_{\dot{H}^{\alpha}} /\|f\|_{L^{2}}\right)^{1 / \alpha}$, to obtain (12).

\section{Proof of the Main Theorem}

The main aim of this section is to study the asymptotic behavior of the global solutions given by Theorem 1 . The proof is inspired from [33].

First, we Take $\theta^{0} \in \mathscr{X}^{1-2 \alpha}$.

Let $\varepsilon>0$, such that $\varepsilon \leq 1 / 8$. For $n \in \mathbb{N}$, put

$$
\mathrm{A}_{n}=\left\{\xi \in \mathbb{R}^{2} ;|\xi| \leq n \text { and }\left|\widehat{\theta}_{0}(\xi)\right| \leq n\right\} \text {. }
$$

We have $\mathscr{F}^{-1}\left(11_{\mathrm{A}_{n}} \cdot \widehat{\theta^{0}}\right)$ converges in $\mathscr{X}^{1-2 \alpha}$ to $\theta^{0}$. Then, there is $n_{0} \in \mathbb{N}$ such that

$$
\left\|\theta^{0}-\mathscr{F}^{-1}\left(11_{\mathrm{A}_{n}} \widehat{\theta}^{0}\right)\right\|_{\mathscr{X}^{1-2 \alpha}} \leq \varepsilon / 4, \quad \forall n \geq n_{0} .
$$

Let $n \geq n_{0}$ be a fixed integer. Put

$$
\begin{aligned}
\theta_{n}^{0} & =\mathscr{F}^{-1}\left(11_{\mathrm{A}_{n}} \cdot \hat{\theta}^{0}\right), \\
w_{n}^{0} & =\theta^{0}-\theta_{n}^{0} .
\end{aligned}
$$

Then,

$$
\begin{aligned}
& \left\|w_{n}^{0}\right\|_{\mathscr{X}^{1-2 \alpha}} \leq(\varepsilon / 4), \\
& \theta_{n}^{0} \in \mathscr{X}^{1-2 \alpha}\left(\mathbb{R}^{2}\right) \cap L^{2}\left(\mathbb{R}^{2}\right) .
\end{aligned}
$$

Consider the system

$\left(\mathrm{QG}_{\alpha}\right)_{n}\left\{\begin{array}{l}\partial_{t} w_{n}+(-\Delta)^{\alpha} w_{n}+u_{w_{n}} \cdot \nabla w_{n}=0, \quad x \in \mathbb{R}^{2}, t>0, \\ w_{n}(0, x)=w_{n}^{0}(x) .\end{array}\right.$

For all $n \geq n_{0}$, we have

$$
\left\|w_{n}^{0}\right\|_{X^{1-2 \alpha}} \leq \frac{\varepsilon}{4} .
$$


Use Theorem 1; then, there exists a unique global solution $w_{n} \in \mathscr{C}\left(\mathbb{R}^{+}, \mathscr{X}^{1-2 \alpha}\left(\mathbb{R}^{2}\right)\right) \cap L^{1}\left(\mathbb{R}^{+}, \mathscr{X}^{1}\left(\mathbb{R}^{2}\right)\right)$.

Furthermore,

$$
\left\|w_{n}(t)\right\|_{X^{1-2 \alpha}}+\left(1-4\left\|w_{n}^{0}\right\|_{\mathscr{X}^{1-2 \alpha}}\right) \int_{0}^{t}\left\|w_{n}(s)\right\|_{X^{1}} \mathrm{~d} s \leq\left\|w_{n}^{0}\right\|_{\mathscr{X}^{1-2 \alpha}}, \quad \forall t \geq 0 .
$$

Moreover,

$$
\left\{\begin{array}{l}
\partial_{t} \theta+(-\Delta)^{\alpha} \theta+u \cdot \nabla \theta=0 \\
\theta(0)=\theta^{0} \in X^{1-2 \alpha}
\end{array}\right.
$$

Put $\theta=\underbrace{\theta-w_{n}}_{\theta_{n}}+w_{n}$. Then, $\theta_{n}$ is a solution of the following system:

$\left\{\begin{array}{l}\partial_{t} \theta_{n}+(-\Delta)^{\alpha} \theta_{n}+u_{\theta_{n}} \cdot \nabla \theta_{n}+u_{\theta_{n}} \cdot \nabla w_{n}+u_{w_{n}} \cdot \nabla \theta_{n}=0, \\ \theta_{n}(0)=\theta_{n}^{0} \in \mathscr{X}^{1-2 \alpha} \cap L^{2} .\end{array}\right.$

By taking the inner product in $L^{2}\left(\mathbb{R}^{2}\right)$ with $\theta_{n}$, we get

$$
\frac{1}{2} \frac{\mathrm{d}}{\mathrm{d} t}\left\|\theta_{n}\right\|_{L^{2}}^{2}+\left\|\theta_{n}\right\|_{\dot{H}^{\alpha}}^{2} \leq\left|\left\langle u_{\theta_{n}} \cdot \nabla w_{n}, \theta_{n}\right\rangle_{L^{2}}\right| .
$$

Then,

$$
\begin{aligned}
& \frac{1}{2} \frac{\mathrm{d}}{\mathrm{d} t}\left\|\theta_{n}\right\|_{L^{2}}^{2}+\left\|\theta_{n}\right\|_{\dot{H}^{\alpha}}^{2} \leq\left|\left\langle u_{\theta_{n}} \cdot \nabla w_{n}, \theta_{n}\right\rangle_{L^{2}}\right| \\
& \leq\left\|u_{\theta_{n}} \cdot \nabla w_{n}\right\|_{L^{2}}\left\|\theta_{n}\right\|_{L^{2}} \\
& \leq C\left\|\mathscr{F}\left(u_{\theta_{n}} \cdot \nabla w_{n}\right)\right\|_{L^{2}}\left\|\theta_{n}\right\|_{L^{2}} \\
& \leq C\left\|\mathscr{F}\left(u_{\theta_{n}}\right) * \mathscr{F}\left(\nabla w_{n}\right)\right\|_{L^{2}}\left\|\theta_{n}\right\|_{L^{2}} .
\end{aligned}
$$

By Young's inequality, we obtain

$\frac{1}{2} \frac{\mathrm{d}}{\mathrm{d} t}\left\|\theta_{n}\right\|_{L^{2}}^{2}+\left\|\theta_{n}\right\|_{\dot{H}^{\alpha}}^{2} \leq C\left\|\mathscr{F}\left(u_{\theta_{n}}\right)\right\|_{L^{2}}\left\|\mathscr{F}\left(\nabla w_{n}\right)\right\|_{L^{1}}\left\|\theta_{n}\right\|_{L^{2}}$

$$
\leq C_{n}\left\|\mathscr{F}\left(\nabla w_{n}\right)\right\|_{L^{1}}\left\|\theta_{n}\right\|_{L^{2}}^{2} .
$$

Therefore,

$$
\frac{1}{2} \frac{d}{d t}\left\|\theta_{n}\right\|_{L^{2}}^{2}+\left\|\theta_{n}\right\|_{\dot{H}^{\alpha}}^{2} \leq C_{n}\left\|w_{n}\right\|_{\mathscr{X}^{1}}\left\|\theta_{n}\right\|_{L^{2}}^{2} .
$$

Integrating with respect to time, we obtain

$\left\|\theta_{n}\right\|_{L^{2}}^{2}+2 \int_{0}^{t}\left\|\theta_{n}\right\|_{\dot{H}^{\alpha}}^{2} \leq\left\|\theta_{n}^{0}\right\|_{L^{2}}^{2}+C_{n} \int_{0}^{t}\left\|w_{n}\right\|_{X^{1}}\left\|\theta_{n}\right\|_{L^{2}}^{2}$.

By Gronwall's lemma, we get

$$
\begin{aligned}
& \left\|\theta_{n}\right\|_{L^{2}}^{2} \leq\left\|\theta_{n}^{0}\right\|_{L^{2}}^{2} \exp \left(C_{n} \int_{0}^{t}\left\|w_{n}\right\|_{\mathscr{X}^{1}}\right) \\
& \leq C_{n}\left\|\theta_{n}^{0}\right\|_{L^{2}}^{2} .
\end{aligned}
$$

Combining (32) and (33), we obtain

$$
\begin{aligned}
\left\|\theta_{n}\right\|_{L^{2}}^{2}+2 \int_{0}^{t}\left\|\theta_{n}\right\|_{\dot{H}^{\alpha}}^{2} \leq\left\|\theta_{n}^{0}\right\|_{L^{2}}^{2}+C_{0}\left\|\theta_{n}^{0}\right\|_{L^{2}}^{2} \int_{0}^{\infty} & \left\|w_{n}\right\|_{X^{1}} \\
& \leq M_{n}
\end{aligned}
$$

Applying Lemma 1 to $\theta_{n}$, we infer

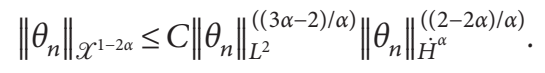

Then,

$$
\left\|\theta_{n}\right\|_{\mathscr{X}^{1-2 \alpha}}^{(\alpha /(1-\alpha))} \leq C\left\|\theta_{n}\right\|_{L^{2}}^{((3 \alpha-2) /(1-\alpha))}\left\|\theta_{n}\right\|_{\dot{H}^{\alpha}}^{2} .
$$

From (33) it follows that

$$
\left\|\theta_{n}\right\|_{\mathscr{X}^{1-2 \alpha}}^{(\alpha /(1-\alpha))} \leq C\left\|\theta_{n}\right\|_{\dot{H}^{\alpha}}^{2} .
$$

Therefore, by integrating in time between 0 and $\infty$, we get

$$
\int_{0}^{\infty}\left\|\theta_{n}\right\|_{X^{1-2 \alpha}}^{(\alpha /(1-\alpha))} \leq C_{0} \int_{0}^{\infty}\left\|\theta_{n}\right\|_{\dot{H}^{\alpha}}^{2} .
$$

Indeed consider the following subset of $[0, \infty[$ :

$$
P_{\varepsilon}=\left\{t \geq 0 ;\left\|\theta_{n}(t)\right\|_{X^{1-2 \alpha}}\left(\mathbb{R}^{2}\right) \geq \frac{\varepsilon}{4}\right\} .
$$

We have

$$
\left\|\theta_{n}(t)\right\|_{\mathscr{X}^{1-2 \alpha}}\left(\mathbb{R}^{2}\right) \geq 1 P_{\varepsilon}(t) \frac{\varepsilon}{4} .
$$

Then, for all $1 / 2<\alpha \leq 1$,

$$
\left\|\theta_{n}(t)\right\|_{X^{1-2 \alpha}}^{(\alpha /(1-\alpha))}\left(\mathbb{R}^{2}\right) \geq \mathbf{1}_{P_{\varepsilon}}(t)\left(\frac{\varepsilon}{4}\right)^{(\alpha /(1-\alpha))} .
$$

Thus,

$$
\int_{0}^{\infty}\left\|\theta_{n}(t)\right\|_{X^{1-2 \alpha}\left(\mathbb{R}^{2}\right)}^{(\alpha /(1-\alpha)} \mathrm{d} t \geq \lambda_{1}\left(P_{\varepsilon}\right)\left(\frac{\varepsilon}{4}\right)^{(\alpha /(1-\alpha))} .
$$

Using the inequality (38), we get $\lambda_{1}\left(P_{\varepsilon}\right)<\infty$ and $\left[0, \infty\left[\backslash P_{\varepsilon} \neq \varnothing\right.\right.$.

Therefore, there exists $t_{0} \in\left[0, \infty\left[\backslash P_{\varepsilon}\right.\right.$. Particularly,

$$
\left\|\theta_{n}\left(t_{0}\right)\right\|_{\mathscr{X}^{1-2 \alpha}}\left(\mathbb{R}^{2}\right)<\frac{\varepsilon}{4}
$$

Now, put

$$
\begin{aligned}
\theta\left(t_{0}\right) & =\underbrace{\theta\left(t_{0}\right)-w_{n}\left(t_{0}\right)}+w_{n}\left(t_{0}\right) \\
& =\theta_{n}\left(t_{0}\right)+w_{n}\left(t_{0}\right) .
\end{aligned}
$$

Thus, by (24), we obtain

$$
\begin{aligned}
& \left\|\theta\left(t_{0}\right)\right\|_{X^{1-2 \alpha}} \leq\left\|\theta\left(t_{0}\right)\right\|_{\mathscr{X}^{1-2 \alpha}}+\left\|w_{n}\left(t_{0}\right)\right\|_{X^{1-2 \alpha}} \\
& \quad \leq \frac{\varepsilon}{4}+\left\|w_{n}^{0}\right\|_{X^{1-2 \alpha}} \\
& \quad \leq \frac{\varepsilon}{4}+\frac{\varepsilon}{4} .
\end{aligned}
$$

Therefore,

$$
\left\|\theta\left(t_{0}\right)\right\|_{\mathscr{X}^{1-2 \alpha}} \leq \frac{\varepsilon}{2} \leq \varepsilon .
$$

Let us consider the following equation: 


$$
\left\{\begin{array}{l}
\partial_{t} \gamma+(-\Delta)^{\alpha} \gamma+u_{\gamma} \cdot \nabla \gamma=0, \quad x \in \mathbb{R}^{2}, t>0 \\
\gamma(0, x)=\gamma^{0}=\theta\left(t_{0}\right)
\end{array}\right.
$$

Using inequality (46) and Theorem 1, we infer that there exists a unique solution $\gamma \in \mathscr{C}\left(\mathbb{R}^{+}, \mathscr{X}^{1-2 \alpha}\left(\mathbb{R}^{2}\right)\right) \cap L^{1}\left(\mathbb{R}^{+}, \mathscr{X}^{1}\left(\mathbb{R}^{2}\right)\right)$ of $(\mathrm{QG})_{\alpha}$ such that

$$
\|\gamma\|_{\mathscr{X}^{1-2 \gamma}}+\left(1-4\left\|\gamma^{0}\right\|_{\mathscr{X}^{1-2 \alpha}}\right) \int_{0}^{t}\|\gamma\|_{\mathscr{X}^{1}} \leq\left\|\gamma^{0}\right\|_{\mathscr{X}^{1-2 \alpha}}, \quad \forall t \geq 0 .
$$

The existence and uniqueness of a solution to the quasigeostrophic equation gives $\forall t \geq 0 \gamma(t)=\theta\left(t_{0}+t\right)$. Then,

$$
\begin{aligned}
& \left\|\theta\left(t_{0}+t\right)\right\|_{\mathscr{X}^{1-2 \alpha}}=\|\gamma(t)\|_{\mathscr{X}^{1-2 \alpha}} \\
& \leq\left\|\gamma^{0}\right\|_{\mathscr{X}^{1-2 \alpha}} \leq \varepsilon .
\end{aligned}
$$

Thus, Theorem 3 is proved.

\section{Data Availability}

No data were used to support this study.

\section{Conflicts of Interest}

The authors declare that there are no conflicts of interest regarding the publication of this paper.

\section{Authors' Contributions}

All authors contributed equally and significantly in writing this article. All authors read and approved the final manuscript.

\section{References}

[1] J. Pedlosky, Geophysical Fluid Dynamics, Springer-Verlag, New York, NY, USA, 2nd edition, 1987.

[2] P. Constantin, A. J. Majda, and E. Tabak, "Formation of strong fronts in the 2-D quasigeostrophic thermal active scalar," Nonlinearity, vol. 7, no. 6, pp. 1495-1533, 1994.

[3] J. Benameur and M. Benhamed, "Global existence of the twodimensional QGE with sub-critical dissipation," Journal of Mathematical Analysis and Applications, vol. 423, no. 2, pp. 1330-1347, 2015.

[4] M. Benhamed, "Global well-posedness and blow-up criterion for the periodic quasi-geostrophic equations in Lei-LinGevrey spaces," Mathematical Methods in the Applied Sciences, vol. 18, pp. 7488-7509, 2017.

[5] H. Dong and D. Du, "Global well-posedness and a decay estimate for the critical dissipative quasi-geostrophic equation in the whole space," Discrete \& Continuous Dynamical Systems - A, vol. 21, no. 4, pp. 1095-1101, 2008.

[6] D. Chae and J. Lee, "Global well-posedness in the supercritical dissipative quasi-geostrophic equations," Communications in Mathematical Physics, vol. 233, no. 2, pp. 297-311, 2003.

[7] T. Iwabuchi and R. Takada, "Global well-posedness and illposedness for the Navier-Stokes equations with the Coriolis force in function spaces of Besov type," Journal of Functional Analysis, vol. 267, no. 5, pp. 1321-1337, 2014.
[8] P. Constantin and J. Wu, "Behavior of solutions of 2D quasigeostrophic equations," SIAM Journal on Mathematical Analysis, vol. 30, no. 5, pp. 937-948, 1999.

[9] P. Constantin, D. Cordoba, and J. Wu, "On the critical dissipative quasi-geostrophic equation," Indiana University Mathematics Journal, vol. 50, no. 1, pp. 97-108, 2001.

[10] L. Caffarelli and A. Vasseur, "Drift diffusion equations with fractional diffusion and the quasi-geostrophic equation," Annals of Mathematics, vol. 171, no. 3, pp. 1903-1930, 2010.

[11] J. Benameur and M. Blel, "Long-time decay to the global solution of the 2D dissipative quasigeostrophic equation," Abstract and Applied Analysis, vol. 2012, Article ID 627813, 12 pages, 2012

[12] J. A. Carrillo and L. C. F. Ferreira, "Convergence towards selfsimilar asymptotic behavior for the dissipative quasi-geostrophic equations," in Self-Similar Solutions of Nonlinear PDE, vol. 74, pp. 95-115, Banach Center Publications, Warsaw, Poland, 2006.

[13] J. A. Carrillo and L. C. F. Ferreira, "Self-similar solutions and large time asymptotics for the dissipative quasi-geostrophic equation," Monatshefte für Mathematik, vol. 151, no. 2, pp. 111-142, 2007.

[14] J. A. Carrillo and L. C. F. Ferreira, “The asymptotic behaviour of subcritical dissipative quasi-geostrophic equations," Nonlinearity, vol. 21, no. 5, pp. 1001-1018, 2008.

[15] A. Córdoba and D. Córdoba, "A maximum principle applied to quasi-geostrophic equations," Communications in Mathematical Physics, vol. 249, no. 3, pp. 511-528, 2004.

[16] C. J. Niche and G. Planas, "Existence and decay of solutions to the dissipative quasi-geostrophic equation with delays," Nonlinear Analysis: Theory, Methods \& Applications, vol. 75, no. 9, pp. 3936-3950, 2012.

[17] C. J. Niche and M. E. Schonbek, "Decay of weak solutions to the 2D dissipative quasi-geostrophic equation," Communications in Mathematical Physics, vol. 276, no. 1, pp. 93-115, 2007.

[18] M. E. Schonbek and T. P. Schonbek, "Asymptotic behavior to dissipative quasi-geostrophic flows," SIAM Journal on Mathematical Analysis, vol. 35, no. 2, pp. 357-375, 2003.

[19] M. Schonbek and T. Schonbek, "Moments and lower bounds in the far-field of solutions to quasi-geostrophic flows," Discrete \& Continuous Dynamical Systems-A, vol. 13, no. 5, pp. 1277-1304, 2005.

[20] M. T. Tun and T. Miyakawa, "On existence and space-time behavior of dissipative 2D quasi-geostrophic flows," Funkcialaj Ekvacioj, vol. 53, no. 2, pp. 169-212, 2010.

[21] Y. Zhou, "Decay rate of higher order derivatives for solutions to the 2-D dissipative quasi-geostrophic flows," Discrete \& Continuous Dynamical Systems- $A$, vol. 14, no. 3, pp. 525-532, 2006.

[22] Y. Zhou, "Asymptotic behaviour of the solutions to the $2 \mathrm{D}$ dissipative quasi-geostrophic flows," Nonlinearity, vol. 21, no. 9, pp. 2061-2071, 2008.

[23] M. Cannone, "Harmonic analysis tools for solving the incompressible Navier-Stokes equations," in Handbook of Mathematical Fluid Dynamics, vol. III, pp. 161-244, NorthHolland, Amsterdam, Netherlands, 2004.

[24] P. G. Lemarie-Rieusset, "Recent developments in the NavierStokes problem," Chapman \& Hall/CRC Research Notes in Mathematics, Vol. 431, Chapman \& Hall/CRC, Boca Raton, FL, USA, 2002.

[25] M. Cannone and G. Karch, "Smooth or singular solutions to the Navier-Stokes system?" Journal of Differential Equations, vol. 197, no. 2, pp. 247-274, 2004. 
[26] Y. L. Jan and A. S. Sznitman, "Stochastic cascades and 3dimensional Navier-Stokes equations," Probability Theory and Related Fields, vol. 109, no. 3, pp. 343-366, 1997.

[27] M. Cannone and G. Wu, "Global well-posedness for NavierStokes equations in critical Fourier-Herz spaces," Nonlinear Analysis: Theory, Methods \& Applications, vol. 75, no. 9, pp. 3754-3760, 2012.

[28] P. Konieczny and T. Yoneda, "On dispersive effect of the Coriolis force for the stationary Navier-Stokes equations," Journal of Differential Equations, vol. 250, no. 10, pp. 38593873, 2011.

[29] Z. Lei and F.-H. Lin, "Global mild solutions of Navier-Stokes equations," Communications on Pure and Applied Mathematics, vol. 64, no. 9, pp. 1297-1304, 2011.

[30] L. C. F. Ferreira and L. S. M. Lima, "Self-similar solutions for active scalar equations in Fourier-Besov-Morrey spaces," Monatshefte für Mathematik, vol. 175, no. 4, pp. 491-509, 2014.

[31] T. Iwabuchi, "Global well-posedness for Keller-Segel system in Besov type spaces," Journal of Mathematical Analysis and Applications, vol. 379, no. 2, pp. 930-948, 2011.

[32] M. F. de Almeida, L. C. F. Ferreira, and L. S. M. Lima, "Uniform global well-posedness of the Navier-Stokes-Coriolis system in a new critical space," Mathematische Zeitschrift, vol. 287, no. 3-4, pp. 735-750, 2017.

[33] I. Gallagher, D. Iftimie, and F. Planchon, "Asymptotics and stability for global solutions to the Navier-Stokes equations," Annales de l'institut Fourier, vol. 53, no. 5, pp. 1387-1424, 2003. 